\title{
Introduction
}

2

3 Autologous chondrocyte implantation (ACI) is a two-stage surgical technique employed to

4 address full thickness, symptomatic chondral lesions. Initially, it involves a cartilage biopsy,

5 isolation and expansion of chondrocytes ex-vivo, and subsequent re-implantation into the

6 damaged joint. Chondrocyte seeded porcine collagen membrane as graft composite is

7 considered to be the next generation ACI techniques, and the cell seeded graft can be fixed to

8 the subchondral bone without suture. Encouraging clinical outcomes using a chondrocyte

9 seeded collagen membrane (matrix-induced ACI - MACI) have been reported in the knee $e^{4,10 \text {, }}$

$1014,24,32,43$ and ankle. ${ }^{8,15,17,27,34}$ With the development of surgical techniques both knee and

11 ankle ACI permit arthroscopic delivery of the scaffold, ${ }^{9}, 12,13,15,16,20,24-26,29,35,40$ which may

12 permit accelerated rehabilitation and minimize the morbidity of arthrotomy including 13 reducing the risk of complications such as adhesions, joint stiffness, excessive pain and 14 scarring.

While a range of surgical options exist for addressing cartilage defects in the shoulder including debridement, microfracture, osteochondral autograft transfer (OATS) and osteochondral allografts, ${ }^{11,18,41}$ published evidence reporting on ACI in the glenohumeral joint is limited to one case report (humeral head) ${ }^{39}$ and one small case series $(n=4),{ }^{5}$ of which three cases were also on the humeral head. We describe the surgical technique and clinical and radiological outcomes to 24 months in a young patient undergoing arthroscopic ACI for a symptomatic cartilage defect on the glenoid.

24 Keywords: autologous chondrocyte implantation; arthroscopic; clinical outcomes; magnetic 25 resonance imaging; shoulder; glenohumeral joint. 
A 25-year-old female nurse presented with pain and functional limitation of her dominant right shoulder, following a direct blow to the anterior aspect of her shoulder from another player during a game of Gaelic football. This resulted in severe pain immediately, though there was no reported sensation of an obvious glenohumeral dislocation or subluxation. Due to persistent discomfort and a painful arc high resolution magnetic resonance imaging (MRI) was undertaken 6 days post-injury, demonstrated a large shear injury to the glenoid articular surface from 9 o'clock to 6 o'clock, with a chondral defect measuring $14 \mathrm{~mm}$ (anteroposterior) by $10 \mathrm{~mm}$ (cranio-caudal). The displaced chondral fragment was visualised in the anterior joint, and the labrum and glenohumeral ligaments were unremarkable, as were other supporting bony and soft tissue structures. The patient was otherwise well, a non-smoker with no other medical history, nor any prior shoulder symptoms or injuries.

First Stage Arthroscopy and Cartilage Harvest

An arthroscopy was performed 14 days post-injury to evaluate the extent of the injury and remove the displaced cartilage fragment, as well as harvest donor cells for ACI. A standard shoulder arthroscopy was performed in the lateral position with a posterior and anterior portal. The loose fragment was found in the anterior inferior joint space (Figure 1A) and was removed and seen to be purely cartilaginous (Figure 1B). This was sent to the laboratory (Orthocell, Perth, Western Australia), where chondrocytes were isolated from the tissue and cultured for approximately 4-8 weeks. At this time, the joint was further evaluated, and the rotator cuff, long head of biceps and humeral articular surface were all normal. The full thickness articular cartilage defect was identified on the glenoid (Figure 2A), while the posterior glenoid chondrolabral junction was torn and mobile, but not grossly unstable. Two 
51 Y knot $1.3 \mathrm{~mm}$ anchors (Conmed Linvatec, Largo FL) were used to stabilize the posterior

52 labrum (Figure 2B). Simple sutures were passed with a Spectrum MVP passer (Conmed

53 Linvatec, Largo FL). This provided a defect with well-defined and stable margins for the

54 second stage graft implantation. Post-operatively the patient was managed in a simple sling

55 for comfort. Range of motion was limited to avoid internal rotation and lifting with this arm

56 was limited to $2 \mathrm{~kg}$ to avoid loading and damaging the margins of the defect.

57

Autologous Chondrocyte Implantation

The second stage re-implantation was performed 8 weeks later. A standard lateral arthroscopy was performed. Posterior and anterior (just above subscapularis in the rotator interval) portals were created and two 8 x $75 \mathrm{~mm}$ DryDoc cannulas (Conmed Linvatec, Largo FL) were inserted. There are no internal valves in these cannulas and the outer caps were removed to allow easy passage of the graft during the dry arthroscopy. A viewing portal was placed high in the rotator interval just below long head of biceps (Figure 3A). The joint was assessed, and fibrous material had partially filled the defect (Figure 3B), which was debrided to subchondral bone with curette (Figure 3C).

Fluid was then drained from the shoulder, and a long metal sucker was used to maintain a dry working environment, ensuring the defect bed was dry. An adrenaline soaked patty was used on the subchondral bone to further dry the area and provide haemostasis. Two vials 71 (approximately 4.91 million cells per $1 \mathrm{ml}$ vial) totalling approximately 9.82 million cells 72 (chondrocytes) were extracted into a $2 \mathrm{ml}$ syringe using a 19 gauge needle, and then dropped 73 intra-operatively onto the porous side of a Type I collagen membrane (size of $3 \times 4 \mathrm{~cm}^{2}$ ). The 74 chondrocytes were at passage 3 when seeded onto the membrane. This collagen membrane was supplied for cell seeding according to the Therapeutic Goods Administration (TGA) 
approved manufacture procedure for Ortho-ACI (Australian Register of Therapeutic Goods listed product code is 289402, https://www.tga.gov.au/artg/artg-id-289402). After a 20 minute incubation period in a small container at room temperature, chondrocytes were integrated into the three-dimensional porous collagen membrane. A surgical pen was employed to mark the membrane to aid in subsequent arthroscopic implantation, and the membrane was then trimmed based on the template made by the foil (from a suture pack) to ensure a good fit without any overhang. Once the membrane shape and size was confirmed, two 2-0 vicryl sutures with a taper needle were passed under the labrum at the edge of the defect with a Spectrum MVP passer (Conmed Linvatec, Largo FL). The suture ends were left long through the anterior and posterior cannulas, with care taken not to cross the sutures. The anterior suture ends with the needles were passed through the graft in the corresponding edge at a similar position. The needles were cut off and a simple knot made to prevent the suture pulling through the seeded chondrocyte membrane.

The seeded chondrocyte membrane graft was then folded in half and passed through the anterior cannula with an arthoscopic grasper, and the vicryl sutures were shortened from the posterior cannula as the graft was introduced. Once in the joint, the graft was unfolded and left loose away from the defect. Fibrin sealant (Tisseel ${ }^{\circledR}$, Baxter International, Illinois, U.S.) was placed in the defect using a long spinal needle through the anterior cannula (Figure 3D). The graft was then placed, using the arthroscopic grasper to guide and pulling on the vicryl sutures to dock the graft, and a silicone foley catheter was then passed through the cannulas from posterior to anterior (Figure 3E). The tip was held with the arthroscopic grasper to control the position of the balloon, and the catheter inflated with normal saline. This was employed to provide an even pressure to the membrane and allowed visualisation of the graft through the balloon. Pressure was maintained for a 30 second period, to ensure the fibrin glue 
101

102

103

104

105

106

107

108

109

110

111

112

113

114

115

116

117

118

119

120

121

122

123

124

125

becomes adherent and provided uniform graft stability. The shoulder was then taken through a range of motion to ensure graft stability. The vicryl suture ends were then cut flush (Figure 3F), and the portals were closed. The shoulder was placed in a simple sling.

\section{Post-operative Management}

The patient underwent a coordinated post-operative rehabilitation program of progressive exercise and graduated loading over 6 months, while further education and advice was provided up until the 12 month time-point. Initially, the patient was immobilized in a sling for six weeks post-operatively during daily activities (removed for rehabilitation exercises). Following passive range of motion (ROM) exercises over the first 1-2 weeks, the out-patient program was initiated at 2-weeks post-surgery and included early scapula positioning and active-assisted (progressing toward active) ROM exercises, strengthening exercises initially employing therabands, cables and pulleys from 3-4 weeks, and progressive open and closed kinetic chain strengthening as tolerated from six weeks onwards.

\section{Clinical Outcome Measures}

Clinical assessment was undertaken pre-surgery and at 3, 6, 12 and 24 months post-surgery, using: 1) the Oxford Shoulder Score (OSS); 2) the Upper Extremity Functional Scale (UEFS) $;^{37} 3$ ) the Disability of the Arm, Shoulder and Hand (DASH) questionnaire; ${ }^{21,22}$ 4) a Visual Analogue Pain Scale assessing the frequency (VAS-F) and severity (VAS-S) of pain; 5) a Global Rating of Change (GRC) scale; ${ }^{23}$ 6) a Patient Satisfaction Questionnaire, and; 7) the Constant Score. ${ }^{7}$ The OSS is a 12-item questionnaire evaluating pain and function, and an improvement of 6 points has been reported as the minimal detectable change (MDC). ${ }^{46}$ The UEFS is a 20-item survey reporting on upper limb function, ${ }^{37}$ and an MDC of 9.4 has been reported. ${ }^{6}$ The DASH is a 30 -item questionnaire evaluating pain, symptoms and physical 
126 function, as well as work and sport capacity, in patients with upper limb musculoskeletal 127 disorders. ${ }^{21,22}$ An MDC for the DASH has been reported as 12.7 points. $^{3}$ The VAS required 128 the patient to rate their pain frequency $(0=$ never, $10=$ constant $)$ and intensity $(0=$ no pain, $12910=$ worst pain imaginable) on a $0-10 \mathrm{~cm}$ sliding scale in the preceding 24 hours. A $1.4 \mathrm{~cm}$ 130 improvement on the VAS has previously been reported as the minimal clinically important 131 change (MCID) in patients undergoing non-operative treatment for rotator cuff disease. ${ }^{44}$

The Constant Shoulder Score ${ }^{7}$ has been validated for total shoulder arthroplasty, ${ }^{1}$ rotator cuff repair, adhesive capsulitis of the shoulder, ${ }^{33}$ and proximal humeral fractures. ${ }^{2}$ The subjective component of the Constant score is allotted a total of 35 points and evaluates patient-reported 136 pain (15 points), as well as how the patient's shoulder condition affects their ability to 137 undertake occupational, leisure and other daily activities (20 points). Active ROM is allotted 138 40 points consisting of forward flexion (10 points), abduction (10 points), functional external rotation (10 points) and internal rotation (10 points). Maximal pain-free isometric shoulder abduction strength in $90^{\circ}$ of shoulder abduction in the scapular plane is allotted 25 points. 141 Finally, a 'total' Constant score was calculated (0-100) by summing the three individual sub142 scales. The MDC for the Constant score has been reported as 18 points for rotator cuff tears. ${ }^{19}$ 143 An 11-point Global Rating of Change (GRC) scale ${ }^{23}$ was employed to evaluate the patient's 144 perceived current status compared to before their surgery, ranging from -5 (very much worse) 145 to 0 (about the same) to 5 (completely recovered). Finally, a patient satisfaction questionnaire 146 was employed to evaluate the patient's level of satisfaction with their surgery overall, as well 147 as their satisfaction with surgery to relieve their shoulder pain, improve their ability to 148 perform normal daily and work activities, improve their ability to return to recreational activities (e.g. swimming, golf) and improve their ability to participate in sporting activities 
150 (e.g. tennis, squash). A categorical tool was employed: $1=$ very satisfied; $2=$ somewhat 151 satisfied; 3 = somewhat dissatisfied; 4 = very dissatisfied.

152

153 Radiological Evaluation

154 High resolution MRI was undertaken pre-surgery and at 4, 12 and 24 months post-surgery, 155 using $1.5 \mathrm{~T}$ and $3 \mathrm{~T}$ clinical scanners (Ingenia, Philips Healthcare, Best, Netherlands). 156 Standardized proton density and T2-weighted fat-saturated images were obtained in coronal157 oblique and sagittal-oblique planes (slice thickness $3-3.5 \mathrm{~mm}$, field of view $14 \mathrm{~cm}$, minimum 158512 matrix in at least one axis). Additional axial proton density fat-saturated images were 159 obtained (slice thickness $3.5 \mathrm{~mm}$, field of view $14 \mathrm{~cm}$, minimum 480 matrix in at least one 160 axis).

161

162 We sought to evaluate eight pertinent parameters of graft repair (graft infill, signal intensity, 163 border integration, surface contour, tissue structure, effusion, subchondral lamina and bone), ${ }^{31}$ 164 following the magnetic resonance observation of cartilage repair tissue (MOCART) scoring 165 system. $^{30,38,45,47}$ The eight defined parameters were each scored from 1-4 (1=poor; 2=fair; $1663=$ =good; 4=excellent) in comparison to the adjacent native cartilage. An MRI composite score 167 was then calculated (scored 0-4) by multiplying each individual score by a weighting factor, ${ }^{38}$ 168 and adding the scores together. MRI evaluation was performed by an independent, 169 experienced musculo-skeletal radiologist. 


\section{Results}

171

172

\section{Clinical Outcome}

173 A significant clinical improvement was seen in all scores over time, with the maximum

174 possible score attained for each patient-reported outcome measure by 24 months post-surgery 175 and all MDCs were exceeded over this time (Table 1). ${ }^{3,6,19,46}$ The patient perceived their 176 shoulder to be fully recovered by 24 months as per the GRC, and the patient was "very 177 satisfied' with the surgery for relieving pain, improving their ability to perform normal daily 178 and work activities, and improving their ability to return to recreational activities. The patient 179 was 'satisfied' with the surgery for improving their ability to participate in sporting activities.

181 Radiographic Outcome

182 MRI demonstrated a progressive infill in regenerative tissue and signal intensity up until 12 183 months, sustained to 24 months. At 24 months, the graft was isointense and full, comparative 184 to the adjacent native cartilage, with a smooth surface and imperceptible border zone 185 integration (Figure 4). As per the MOCART scoring system, graft infill was scored as 0 (poor $186=$ subchondral bone exposed), 3 ( $\operatorname{good}=>50 \%$ height of adjacent cartilage), 4 (complete $=$ 187 level with the adjacent native cartilage) and 4, at pre-surgery and 4, 12 and 24 months post188 surgery, respectively. The overall MOCART MRI composite score was graded as 1.35, 2.80, 1893.95 and 4.00 (out of 4.00) at the aforementioned time points. 


\section{Discussion}

This single case study presents a successful outcome following third-generation ACI performed on the glenoid, with improved clinical and radiological outcomes to 24 months

194 post-surgery. While outcomes are encouraging following ACI in other joints such as the knee 195 and ankle, ${ }^{4,8,10,14,15,17,24,27,32,34,43}$ published evidence of its use in the glenohumeral joint is 196 limited to one case report (humeral head) ${ }^{39}$ and one small $(n=4)$ case series, ${ }^{5}$ of which three cases were also on the humeral head.

199 The single case by Romeo et al. ${ }^{39}$ employed first generation periosteal-covered ACI, though 200 no specific clinical outcomes were reported apart from the manuscript stating that ROM was 201 full at 12 months post-surgery with no further patient complaints of pain at rest or with weather changes. Furthermore, first-generation techniques are more surgically complex, may result in extensive micro-trauma and cell leakage, have donor morbidity from periosteal 204 harvest, increased rates of graft hypertrophy and require more exposure for suturing the cover, 205 therefore not permitting arthroscopic surgical techniques. ${ }^{28,36}$ Buchman et al. $^{5}$ further reported 206 on four cases undergoing ACI (autologous chondrocyte transplantation collagen membrane 207 seeding - ACT-C) in the glenohumeral joint (3 humeral head, 1 glenoid), with encouraging 208 clinical outcomes reported at a mean follow up of 41.3 \pm 24.9 months (range 11-71 months).

210 In determining the best surgical option for cartilage defects in the glenohumeral joint, a 211 number of factors must be considered including defect location, size, depth and containment, 212 as well as concurrent pathology that must be addressed at the time of surgery. ${ }^{41}$ Alternative 213 surgical procedures for this patient may have included microfracture (with or without 214 augmentation) or OATS. In a randomized controlled trial, Saris et al. ${ }^{42}$ demonstrated that by 
21524 months post-surgery the use of matrix-applied characterized autologous cultured 216 chondrocytes was clinically and statistically significantly better than microfracture, albeit this 217 was in the differently behaving knee joint and in symptomatic cartilage defects $\geq 3 \mathrm{~cm}^{2}$. While 218 lower compressive loads may occur across the glenohumeral joint (compared with the knee 219 and ankle), high shear stress may occur given the large ROM and speed of rotation that occurs 220 in this joint, and ACI was deemed the most appropriate option when considering her age and 221 requirement for a more sustainable tissue repair. Furthermore, given the relative scarcity of 222 published evidence on glenohumeral ACI and associated rehabilitation protocols, the protocol 223 followed was developed based on clinical experience in treating other shoulder pathologies, 224 combined with knowledge gained from treating patients embarking on ACI in the knee and 225 ankle. 
ACI in the glenohumeral joint

\section{Conclusion}

228 The management of focal cartilage defects in the glenohumeral joint remains a challenge.

229 This case study presents a successful outcome following arthroscopic third-generation ACI 230 performed on the glenoid, combined with a structured rehabilitation protocol. Improved 231 clinical outcomes and a high level of patient satisfaction was observed to 24 months post232 surgery, with evidence of regenerative tissue repair sustained to 24 months, similar in 233 characteristics to the adjacent native glenoid articular cartilage. Larger case series are required 234 to better evaluate whether ACI can provide a good therapeutic option for patients presenting 235 with symptomatic cartilage defects in the glenohumeral joint. 


\section{References}

237

2381 Angst F, Pap G, Mannion AF, Herren DB, Aeschlimann A, Schwyzer HK et al. 239 Comprehensive assessment of clinical outcome and quality of life after total shoulder 240 arthroplasty: usefulness and validity of subjective outcome measures. Arthritis Rheum 2004;51:819-828. 10.1002/art.20688

2422 Baker P, Nanda R, Goodchild L, Finn P, Rangan A. A comparison of the Constant and Oxford shoulder scores in patients with conservatively treated proximal humeral fractures. J Shoulder Elbow Surg 2008;17:37-41. 10.1016/j.jse.2007.04.019

Beaton DE, Davis AM, Hudak P, McConnell S. The DASH (Disabilities of the Arm, Shoulder and Hand) outcome measure: What do we know about it now? J Hand Ther 2001;6:109-118. 10.1177/175899830100600401

Behrens P, Bitter T, Kurz B, Russlies M. Matrix-associated autologous chondrocyte transplantation/implantation (MACT/MACI) - 5-year follow-up. Knee 2006;13:194202. 10.1016/j.knee.2006.02.012

2515 Buchmann S, Salzmann GM, Glanzmann MC, Wortler K, Vogt S, Imhoff AB. Early clinical and structural results after autologous chondrocyte transplantation at the glenohumeral joint. J Shoulder Elbow Surg 2012;21:1213-1221. 10.1016/j.jse.2011.07.030

255

6 Chesworth BM, Hamilton CB, Walton DM, Benoit M, Blake TA, Bredy H et al. Reliability and validity of two versions of the upper extremity functional index. Physiother Can 2014;66:243-253. 10.3138/ptc.2013-45

2587 Constant CR, Gerber C, Emery RJ, Sojbjerg JO, Gohlke F, Boileau P. A review of the Constant score: modifications and guidelines for its use. J Shoulder Elbow Surg 2008;17:355-361. 10.1016/j.jse.2007.06.022 
2618 Dixon S, Harvey L, Baddour E, Janes G, Hardisty G. Functional outcome of matrix262 associated autologous chondrocyte implantation in the ankle. Foot Ankle Int $263 \quad 2011 ; 32: 368-374.10 .3113 /$ FAI.2011.0368

2649 Ebert JR, Fallon M, Wood DJ, Janes GC. A Prospective Clinical and Radiological 265 Evaluation at 5 Years After Arthroscopic Matrix-Induced Autologous Chondrocyte 266 Implantation. Am J Sports Med 2017;45:59-69. 10.1177/0363546516663493

26710 Ebert JR, Robertson WB, Woodhouse J, Fallon M, Zheng MH, Ackland T et al. 268 Clinical and magnetic resonance imaging-based outcomes to 5 years after matrix269 induced autologous chondrocyte implantation to address articular cartilage defects in 270 the knee. Am J Sports Med 2011;39:753-763. 10.1177/0363546510390476

$271 \quad$ Elser F, Braun S, Dewing CB, Millett PJ. Glenohumeral joint preservation: current options for managing articular cartilage lesions in young, active patients. Arthroscopy 2010;26:685-696. 10.1016/j.arthro.2009.10.017

274 Erggelet C, Sittinger M, Lahm A. The arthroscopic implantation of autologous chondrocytes for the treatment of full-thickness cartilage defects of the knee joint. Arthroscopy: Arthroscopy 2003;19:108-110. 10.1053/jars.2003.50025

27713 Ferruzzi A, Buda R, Faldini C, Vannini F, Di Caprio F, Luciani D et al. Autologous chondrocyte implantation in the knee joint: open compared with arthroscopic technique. Comparison at a minimum follow-up of five years. J Bone Joint Surg Am 2008;90 Suppl 4:90-101. 10.2106/JBJS.H.00633

28114 Genovese E, Ronga M, Angeretti MG, Novario R, Leonardi A, Albrizio M et al. Matrix-induced autologous chondrocyte implantation of the knee: mid-term and longterm follow-up by MR arthrography. Skeletal Radiol 2010;40:47-56. 10.1007/s00256010-0939-8 
28515 Giannini S, Buda R, Ruffilli A, Cavallo M, Pagliazzi G, Bulzamini MC et al. Arthroscopic autologous chondrocyte implantation in the ankle joint. Knee surgery, sports traumatology, arthroscopy : official journal of the ESSKA 2014;22:1311-1319. $10.1007 / \mathrm{s} 00167-013-2640-7$

28

16 Giannini S, Buda R, Vannini F, Di Caprio F, Grigolo B. Arthroscopic Autologous Chondrocyte Implantation in Osteochondral Lesions of the Talus: Surgical Technique and Results. Am J Sports Med 2008. 10.1177/0363546507312644

29217 Giza E, Sullivan M, Ocel D, Lundeen G, Mitchell ME, Veris L et al. Matrix-induced autologous chondrocyte implantation of talus articular defects. Foot Ankle Int 2010;31:747-753. 10.3113/FAI.2010.0747

29518 Gross CE, Chalmers PN, Chahal J, Van Thiel G, Bach BR, Jr., Cole BJ et al. Operative treatment of chondral defects in the glenohumeral joint. Arthroscopy 2012;28:1889-1901. 10.1016/j.arthro.2012.03.026

29819 Henseler JF, Kolk A, van der Zwaal P, Nagels J, Vliet Vlieland TP, Nelissen RG. The minimal detectable change of the Constant score in impingement, full-thickness tears, and massive rotator cuff tears. J Shoulder Elbow Surg 2015;24:376-381. 10.1016/j.jse.2014.07.003

30220 Ibarra C, Izaguirre A, Villalobos E, Masri M, Lombardero G, Martinez V et al. Follow-up of a new arthroscopic technique for implantation of matrix-encapsulated autologous chondrocytes in the knee. Arthroscopy 2014;30:715-723. 10.1016/j.arthro.2014.02.032

30621 Jester A, Harth A, Germann G. Measuring levels of upper-extremity disability in 307 employed adults using the DASH Questionnaire. J Hand Surg Am 2005;30:1074 e1071-1074 e1010. 10.1016/j.jhsa.2005.04.009 
22 Jester A, Harth A, Wind G, Germann G, Sauerbier M. Disabilities of the arm, shoulder and hand (DASH) questionnaire: Determining functional activity profiles in patients with upper extremity disorders. J Hand Surg $\operatorname{Br}$ 2005;30:23-28. 10.1016/j.jhsb.2004.08.008

23 Kamper S. Global Rating of Change scales. Aust J Physiother 2009;55:289. 10.1016/S0004-9514(09)70015-7

31524 Kon E, Di Martino A, Filardo G, Tetta C, Busacca M, Iacono F et al. Secondgeneration autologous chondrocyte transplantation: MRI findings and clinical correlations at a minimum 5-year follow-up. Eur J Radiol 2010. 10.1016/j.ejrad.2010.04.002

25 Kon E, Filardo G, Berruto M, Benazzo F, Zanon G, Della Villa S et al. Articular Cartilage Treatment in High-Level Male Soccer Players: A Prospective Comparative Study of Arthroscopic Second-Generation Autologous Chondrocyte Implantation Versus Microfracture. Am J Sports Med 2011. 10.1177/0363546511420688

26 Kon E, Gobbi A, Filardo G, Delcogliano M, Zaffagnini S, Marcacci M. Arthroscopic second-generation autologous chondrocyte implantation compared with microfracture for chondral lesions of the knee: prospective nonrandomized study at 5 years. The Am J Sports Med 2009;37:33-41. 10.1177/0363546508323256

32727 Kreulen C, Giza E, Walton J, Sullivan M. Seven-Year Follow-up of Matrix-Induced Autologous Implantation in Talus Articular Defects. Foot Ankle Spec 2017:1938640017713614. 10.1177/1938640017713614

33028 Kreuz PC, Steinwachs M, Erggelet C, Krause SJ, Ossendorf C, Maier D et al. Classification of graft hypertrophy after autologous chondrocyte implantation of fullthickness chondral defects in the knee. Osteoarthritis Cartilage 2007;15:1339-1347. 10.1016/j.joca.2007.04.020 
33429 Marcacci M, Kon E, Zaffagnini S, Filardo G, Delcogliano M, Neri MP et al. Arthroscopic second generation autologous chondrocyte implantation. Knee Surg Sports Traumatol Arthrosc 2007;15:610-619. 10.1007/s00167-006-0265-9

33730 Marlovits S, Singer P, Zeller P, Mandl I, Haller J, Trattnig S. Magnetic resonance observation of cartilage repair tissue (MOCART) for the evaluation of autologous chondrocyte transplantation: Determination of interobserver variability and correlation to clinical outcome after 2 years. Eur J Radiol 2006;57:16-23. 10.1016/j.ejrad.2005.08.007

34231 Marlovits S, Striessnig G, Resinger CT, Aldrian SM, Vecsei V, Imhof H et al. Definition of pertinent parameters for the evaluation of articular cartilage repair tissue with high-resolution magnetic resonance imaging. Eur J Radiol 2004;52:310-319. 10.1016/j.ejrad.2004.03.014

34632 Nehrer S, Dorotka R, Domayer S, Stelzeneder D, Kotz R. Treatment of full-thickness chondral defects with hyalograft $\mathrm{C}$ in the knee: a prospective clinical case series with 2 to 7 years' follow-up. Am J Sports Med 2009;37 Suppl 1:81S-87S. $10.1177 / 0363546509350704$

35033 Othman A, Taylor G. Is the constant score reliable in assessing patients with frozen shoulder? 60 shoulders scored 3 years after manipulation under anaesthesia. Acta Ortho Scand 2004;75:114-116. 10.1080/00016470410001708230

35334 Pagliazzi G, Vannini F, Battaglia M, Ramponi L, Buda R. Autologous Chondrocyte Implantation for Talar Osteochondral Lesions: Comparison Between 5-Year FollowUp Magnetic Resonance Imaging Findings and 7-Year Follow-Up Clinical Results. J Foot Ankle Surg 2017. 10.1053/j.jfas.2017.05.013 
35735 Petersen W, Zelle S, Zantop T. Arthroscopic implantation of a three dimensional scaffold for autologous chondrocyte transplantation. Arch Orthop Trauma Surg 2008;128:505-508. 9-year outcome after autologous chondrocyte transplantation of the knee. Clin Orthop 2000:212-234. 10.1097/00003086-200005000-00020

36337 Pransky G, Feuerstein M, Himmelstein J, Katz JN, Vickers-Lahti M. Measuring functional outcomes in work-related upper extremity disorders. Development and validation of the Upper Extremity Function Scale. J Occup Environ Med 1997;39:1195-1202. 10.1097/00043764-199712000-00014

36738 Robertson WB, Fick D, Wood DJ, Linklater JM, Zheng MH, Ackland TR. MRI and clinical evaluation of collagen-covered autologous chondrocyte implantation (CACI) at two years. Knee 2007;14:117-127. 10.1016/j.knee.2006.11.009

39 Romeo AA, Cole BJ, Mazzocca AD, Fox JA, Freeman KB, Joy E. Autologous chondrocyte repair of an articular defect in the humeral head. Arthroscopy 2002;18:925-929. 10.1053/jars.2002.36144

40 Ronga M, Grassi FA, Bulgheroni P. Arthroscopic autologous chondrocyte implantation for the treatment of a chondral defect in the tibial plateau of the knee.

37641 Saltzman BM, Leroux T, Cole BJ. Management and Surgical Options for Articular Defects in the Shoulder. Clin Sports Med 2017;36:549-572. 10.1016/j.csm.2017.02.009

42 Saris D, Price A, Widuchowski W, Bertrand-Marchand M, Caron J, Drogset JO et al. Matrix-Applied Characterized Autologous Cultured Chondrocytes Versus 
Microfracture: Two-Year Follow-up of a Prospective Randomized Trial. The Am J Sports Med 2014;42:1384-1394. 10.1177/0363546514528093

38343 Saris DB, Vanlauwe J, Victor J, Almqvist KF, Verdonk R, Bellemans J et al. Treatment of symptomatic cartilage defects of the knee: characterized chondrocyte implantation results in better clinical outcome at 36 months in a randomized trial compared to microfracture. Am J Sports Med 2009;37 Suppl 1:10S-19S. $10.1177 / 0363546509350694$

44 Tashjian RZ, Deloach J, Porucznik CA, Powell AP. Minimal clinically important differences (MCID) and patient acceptable symptomatic state (PASS) for visual analog scales (VAS) measuring pain in patients treated for rotator cuff disease. J Shoulder Elbow Surg 2009;18:927-932. 10.1016/j.jse.2009.03.021

39245 Trattnig S, Pinker K, Krestan C, Plank C, Millington S, Marlovits S. Matrix-based autologous chondrocyte implantation for cartilage repair with HyalograftC: two-year follow-up by magnetic resonance imaging. Eur J Radiol 2006;57:9-15. S0720048X(05)00287-1 [pii] 10.1016/j.ejrad.2005.08.006

39646 van Kampen DA, Willems WJ, van Beers LW, Castelein RM, Scholtes VA, Terwee CB. Determination and comparison of the smallest detectable change (SDC) and the minimal important change (MIC) of four-shoulder patient-reported outcome measures (PROMs). J Orthop Surg Res 2013;8:40. 10.1186/1749-799X-8-40

40047 Welsch GH, Mamisch TC, Zak L, Blanke M, Olk A, Marlovits S et al. Evaluation of 401 cartilage repair tissue after matrix-associated autologous chondrocyte transplantation using a hyaluronic-based or a collagen-based scaffold with morphological MOCART scoring and biochemical T2 mapping: preliminary results. Am J Sports Med 
ACI in the glenohumeral joint

\section{Table Legends}

407

408 Table I. Overview of pre- and post-operative clinical outcomes. 
411 Figure 1. Identification of the loose fragment in the anterior inferior joint space at the time of

412 first stage arthroscopic surgery (A), which was seen to be purely cartilaginous (B).

413

414 Figure 2. Identification of the full thickness glenoid defect (A), with subsequent stabilization 415 of the posterior labrum (B).

416

417 Figure 3. Sequence demonstrating the second-stage surgical implantation procedure, 418 including (A) the viewing portal placed high in the rotator interval just below the long head of 419 biceps, (B) identification of the partially filled defect with fibrous material at the time of 420 second-stage implantation, (C) with subsequent debridement of tissue, (D) introduction of the 421 fibrin sealant via a long spinal needle through the anterior cannula, (E) placement of the graft 422 and introduction of the silicone foley catheter which was instilled with saline, and (F) final 423 appearance of the graft.

425 Figure 4. Coronal T2-weighted fat saturated images, demonstrating: (A) pre-operative image 426 of the full-thickness glenoid chondral defect, (B) at 4 months following surgery the chondral 427 graft is hyperintense to native cartilage and chondral fill is between $50-100 \%$ of the native 428 cartilage thickness, and (C) at 12 months and (D) 24 months following surgery the chondral 429 graft is isointense and approximates the thickness of native cartilage. 